VEGA, F.V.A.; BOVI, M.L.A.; SPIERING, S.H.; GODOY JÚNIOR, G. Relações alométricas para estimativa da fitomassa aérea em pupunheira. Horticultura Brasileira, Brasília, v.22, n.1, p. 104-108, jan-mar 2004.

\title{
Relações alométricas para estimativa da fitomassa aérea em pupunheira
}

Fernando Vinicio A. Vega; Marilene L. A. Bovi; Sandra Heiden Spiering; Gentil Godoy Júnior

Instituto Agronômico, C. Postal 28, 13001-970 Campinas-SP; E-mail: fernandoarmas@yahoo.com.br

\section{RESUMO}

A estimativa da fitomassa aérea da pupunheira (Bactris gasipaes Kunth) por meio de relações alométricas tem aplicação teórica e prática, sendo essencial em estudos de fisiologia de crescimento, bem como para identificar respostas e predizer a produção. No presente trabalho foram avaliadas diferentes equações buscando o melhor ajuste alométrico representativo da fitomassa da pupunheira cultivada para a produção de palmito. Foram utilizadas palmeiras inermes, da raça Putumayo, em diferentes estádios de desenvolvimento, cultivadas em Ubatuba (SP) no espaçamento de $2 \times 1 \mathrm{~m}$. Selecionaram-se 117 plantas, com alturas entre 0,22 e 5,04 m e diâmetros entre 2,23 e 27,06 cm. Medidas diretas, relacionadas ao crescimento, foram realizadas antes do corte. Em seguida as plantas foram separadas em diferentes partes estruturais, sendo medidas, pesadas e secas, obtendo-se a massa da matéria seca. Os dados foram submetidos à análise de regressão e ajuste de equações, tendo como variáveis independentes os caracteres facilmente mensuráveis e não destrutivos. A fitomassa da pupunheira pode ser estimada de forma precisa a partir de equações simples, valendo-se de relações alométricas. A altura da haste principal, medida do solo até a inserção da folha +1 , foi o caráter preditório indireto ideal para estimar a fitomassa de pupunheiras em cultivo comercial. Identificou-se também que, do estádio de implantação ao início de colheita de palmito, a contribuição dos perfilhos para a fitomassa aérea total é pequena e pode ser desprezada.

Palavras-chave: Bactris gasipaes, biomassa, crescimento, perfilhos, pupunha.

\section{ABSTRACT \\ Peach palm biomass estimates based on allometric relationships}

Biomass estimates based on allometric relationships have theoretical and practical application. These data are useful tools in growth analysis experiments and yield prediction. Several equations were studied to define the best allometric fit to peach palm grown for heart-of-palm purpose. Spineless peach palms (Bactris gasipaes Kunth), from Putumayo landrace, were utilized. The experiment, in a $2 \times 1 \mathrm{~m}$ planting density, was conducted at Ubatuba, São Paulo State, Brazil. Where 117 plants were selected, ranging from 0.22 to $5.04 \mathrm{~m}$ of main stem height, and from 2.23 to $27.06 \mathrm{~cm}$ of main stem diameter. Direct growth measurements were taken before plant harvesting. Harvested material was separated in different structural components and weighted before and after drying. Regression analyses were performed and different equations were applied to data, having as independent variables the traits easily measured. Peach palm biomass can be precisely estimated by allometric relationships. Main stem height, measured from ground level until first leaf insertion, was the ideal trait to indirectly estimate biomass in peach palm commercial cultivation. The contribution of the affshots biomass to the total above ground biomass, from planting to first harvesting is very low and can be neglected.

Keywords: Bactris gasipaes, growth, heart-of-palm, pejibaye, offshoot.

(Recebido para publicação em 11 de março de 2003 e aceito em 10 de outubro de 2003)

\begin{abstract}
$\mathrm{A}$ pupunheira (Bactris gasipaes Kunth), palmeira nativa da América tropical, apresenta características de precocidade, rusticidade e perfilhamento, como atributos ideais para a produção de palmito (Bovi, 1998a). Produto de largo consumo, o palmito é constituído basicamente pelo meristema apical e um número variável de folhas internas, ainda não plenamente desenvolvidas e imbricadas, sendo envolto e protegido pela bainha das folhas adultas mais externas (Ferreira $e t$ al., 1976; Bovi, 1998a). Portanto, um desenvolvimento foliar adequado é a condição principal para obter resultado satisfatório na produção de palmito. Pelas características próprias do cultivo, com colheitas freqüentes e escalonadas em função do desenvolvimento das hastes, as plantas apresentam-
\end{abstract}

se em permanente estádio vegetativo (Clement, 1995) e a velocidade com que a fitomassa inicial se acumula e é reposta novamente após cada colheita, torna-se um indicador da produtividade e da vida econômica da cultura (Clement e Bovi, 2000). Por esse motivo, a pupunheira vem sendo alvo de vários estudos na área de fisiologia do crescimento e da produção, bem como de suas inter-relações com as condições climáticas e, principalmente, com a nutrição mineral e orgânica.

A análise de crescimento permite conhecer as diferenças funcionais e estruturais entre plantas e estimar o acúmulo de fitomassa, de forma a identificar respostas à aplicação de diferentes tratamentos, assim como prever a produção. Em plantas anuais, a análise de crescimento é feita, geralmente, por meio do método direto, usando-se amostras destrutivas, colhidas ao longo do ciclo da planta (Benincasa, 1988). Por meio da obtenção da massa total da planta, ou de seus diversos componentes, estima-se o acúmulo de fitomassa ao longo do tempo. Já em plantas perenes, principalmente em plantios comerciais ou em estudos com plantas de grande valor econômico, o método indireto, ou não destrutivo, é o mais indicado, devido principalmente às dificuldades logísticas da obtenção de dados (Benincasa, 1988; Ares e Fownes, 2000). Esse método consiste na aplicação de análise dimensional, também conhecida como alometria (Kira e Shidei, 1967). A base do método é o estabelecimento de parâmetros que indiquem ou expressem, em forma alométrica, o estado atual de uma planta. Para isso al- 
guns autores propõem um número variado de equações baseado em medidas diretas como altura, diâmetro, comprimento e número de estruturas (perfilhos, ramificações, entre outros) que possam estimar, com suficiente precisão, a massa ou o desenvolvimento da planta como um todo. Não obstante, deve ser ressaltado que para o estabelecimento dessas relações alométricas há necessidade de serem efetuadas análises destrutivas. No entanto, essas são feitas em amostragem, de preferência em algumas plantas do próprio experimento, ou pelo menos em plantas da mesma origem genética, em estádios ontogenéticos semelhantes, e cultivadas nas mesmas condições agrobioclimáticas.

Equações que visam estimar a fitomassa da planta inteira ou de seus componentes foram desenvolvidas por alguns autores para a pupunheira, mas, invariavelmente, se referem ao estado adulto da planta, visto que anteriormente essa espécie era apenas usada para a produção de frutos (Clement et al., 1990; Szott et al., 1993). Estimativas envolvendo plantas mais jovens foram publicadas por Jongschaap (1993) e Clement (1995), representando o período de formação de mudas e a fase inicial de implantação a campo. Apenas recentemente foi desenvolvido estudo com plantas destinadas a palmito (Ares et al., 2002b), mas, mesmo assim, o genótipo utilizado foi da raça UtilisTucurrique, material com espinhos, bastante diferente do que vem sendo cultivado no Brasil desde 1990 (Bovi, 1998b). Embora os trabalhos anteriores forneçam informações valiosas sobre as relações alométricas em pupunheira, reconhece-se também que são de aplicação limitada. De acordo com Clement e Bovi (2000), relações alométricas precisam ser validadas para cada germoplasma e ambiente (latitude, altitude, solo, adubação, irrigação, densidade, etc), pois a fitomassa possui plasticidade e baixa herdabilidade (Clement, 1995; Lucchesi, 1987).

Este trabalho teve como objetivo avaliar diferentes tipos de equações, tentando encontrar o melhor ajuste alométrico para a estimativa da fitomassa aérea da pupunheira quando cultivada para a produção de palmito.

\section{MATERIAL E MÉTODOS}

Foram utilizadas pupunheiras (Bactris gasipaes Kunth) inermes, da raça Putumayo, em diferentes estádios de desenvolvimento. As plantas foram cultivadas no espaçamento de $2 \times 1 \mathrm{~m}$ (5000 plantas ha $\mathrm{a}^{-1}$ ), densidade recomendada para a produção de palmito (Bovi, 1998a). O cultivo foi realizado na E.E. do Instituto Agronômico de Campinas, localizada em Ubatuba $\left(23^{\circ} 27^{\prime} \mathrm{S}\right.$,

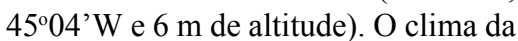
região é "Cfa", pela classificação de Köppen, tropical, quente e úmido, com pluviosidade anual normal de $2841 \mathrm{~mm}$, evapotranspiração potencial normal de $992 \mathrm{~mm}$, excedente normal de $1849 \mathrm{~mm}$, temperatura média anual de $20,8^{\circ} \mathrm{C}$ e déficit hídrico nulo. $\mathrm{O}$ solo da área do cultivo é arenoso, classificado como Aluvial álico (Udifluvent), com boa drenagem. Detalhes da composição granulométrica e química do solo encontram-se em Vega (2003).

Foram selecionadas 117 plantas, sendo 82 hastes principais (planta mãe) e 35 perfilhos, abrangendo todos os estádios de crescimento representativos tanto de lavoura bem manejada para a produção de palmito, quanto daquela onde o corte não é freqüente ou são deixadas algumas plantas matrizes para produção de frutos. Seguindo os procedimentos e recomendações fornecidos por Benincasa (1988) e Clement e Bovi (2000), foram realizadas as seguintes medidas na haste principal: altura até a inserção da folha $+1(\mathrm{~m})$, perímetro à altura do colo da planta $(\mathrm{m})$ e número de folhas. Após essas medidas, as hastes foram cortadas na base (rente ao solo), separando-se as diferentes partes estruturais da planta para serem pesadas e medidas individualmente. $\mathrm{O}$ material vegetal foi colocado em estufa a $60^{\circ} \mathrm{C}$ até obtenção de peso constante, pesando-se novamente todas as amostras. A área foliar $\left(\mathrm{m}^{2}\right)$ foi estimada pelo método de uso de áreas conhecidas de lâminas foliares, descrito por Benincasa (1988).

Foi feita análise preliminar dos dados visando caracterizar a população amostrada para cada caráter estudado. Além da média, calculou-se o desvio padrão, o coeficiente e a amplitude de variação e o intervalo de confiança da média a $5 \%$ de probabilidade (Steel e Torrie, 1980). Foi realizada ainda, análise de regressão e ajuste de equações, tendo como variável dependente os caracteres de natureza destrutiva e como independente os facilmente mensuráveis e não destrutivos (Hyams, 1997). As equações foram selecionadas levandose em conta o alto grau de correlação entre variáveis (coeficiente de determinação), a comparação 1:1 dos dados estimados na equação com os dados reais das plantas, e o significado biológico.

Visando identificar a contribuição dos perfilhos para a fitomassa total durante a primeira fase da cultura (plantio até a primeira colheita de palmito) foram contadas e medidas todas as hastes principais, bem como todos os perfilhos de plantas de um experimento situado no mesmo local com 12 meses a campo. A fitomassa de ambos (haste principal e perfilhos) foi estimada usando-se as equações mais adequadas, definidas na etapa anterior.

\section{RESULTADOS E DISCUSSÃO}

A população em estudo foi composta por 82 plantas-mãe com altura da haste principal variando de 0,22 a $5,04 \mathrm{~m}$ (média de 1,75 m, desvio padrão (DP) de $0,94 \mathrm{~m}$, coeficiente de variação $(\mathrm{CV})$ de $53,72 \%$ e intervalo de confiança (IC) de $0,21 \mathrm{~m})$, e de 0,07 e $0,85 \mathrm{~m}$ de perímetro (média de $0,38 \mathrm{~m}, \mathrm{DP}$ de $0,14 \mathrm{~m}$, $\mathrm{CV}$ de $37,50 \%$ e IC de $0,03 \mathrm{~m}$ ), o que corresponde a diâmetros de 2,23 a 27,06 $\mathrm{cm}$, respectivamente. Da mesma forma, para os 35 perfilhos amostrados, as alturas variaram entre 0,05 e $0,58 \mathrm{~m}$ (média de $0,25 \mathrm{~m}$, DP de $0,16 \mathrm{~m}, \mathrm{CV}$ de $63,52 \%$ e IC de $0,06 \mathrm{~m}$ ), e os perímetros entre 0,06 e $0,26 \mathrm{~m}$ (média $0,14 \mathrm{~m}$, DP de $0,06 \mathrm{~m}, \mathrm{CV}$ de $43,39 \%$ e IC de $0,21 \mathrm{~m}$ ), equivalentes a $1,91-8,28 \mathrm{~cm}$ de diâmetro, respectivamente. O número de folhas verdes da haste principal variou de 3 a 18 (média de 7,17, DP de 2,78, CV de $98,66 \%$ e IC de 0,62 ), enquanto a área foliar estimada dessa mesma haste esteve entre 0,69 e $100,09 \mathrm{~m}^{2}$, com média de $14,32 \mathrm{~m}^{2}$ e CV de $98,66 \%$.

O CV variou entre $37,50 \%$ (para o perímetro da haste principal) e $98,66 \%$ 

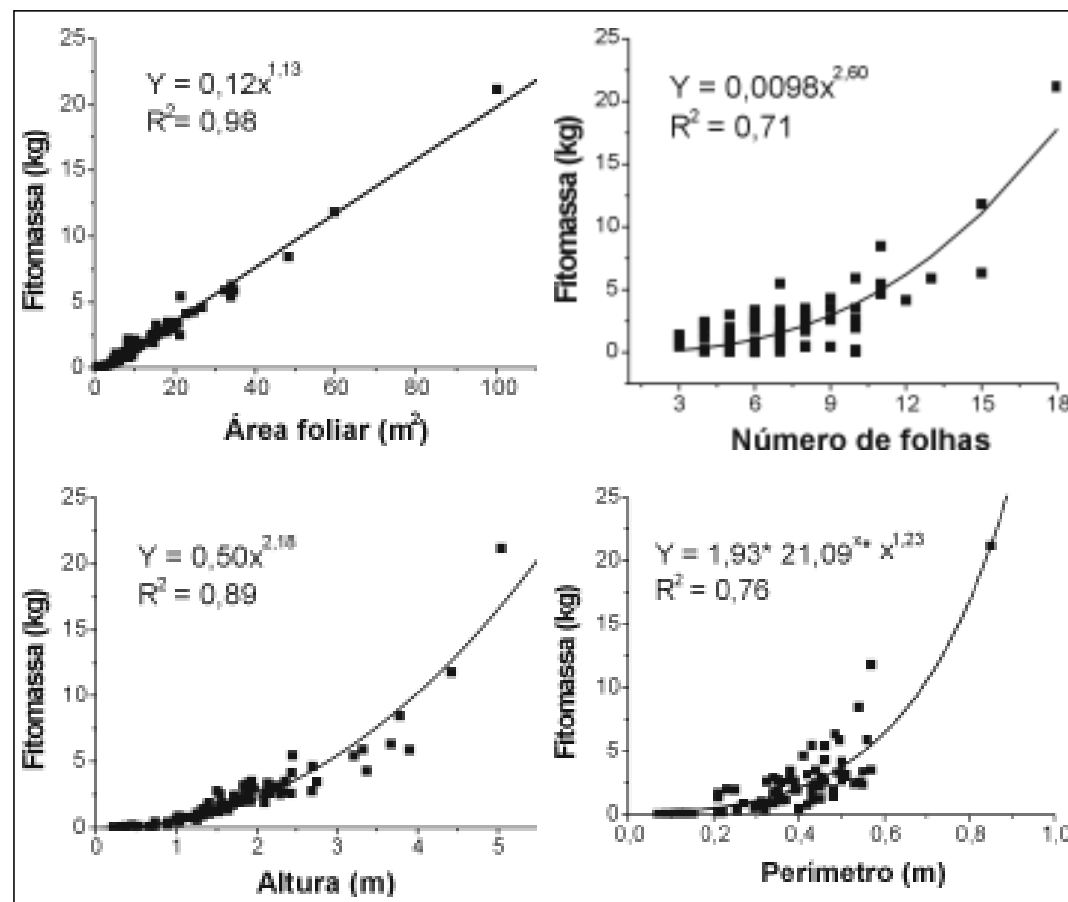

Figura 1. Ajustes de curva para estimativa da fitomassa da pupunheira (kg planta-1), com base em matéria seca, em função da área foliar estimada $\left(\mathrm{m}^{2}\right)$, número de folhas, altura até a inserção da folha $+1(\mathrm{~m})$ e perímetro da planta na região do colo (m). Ubatuba, IAC, 2002.

(correspondente à estimativa da área foliar total da haste principal). Esses resultados indicam que além da variabilidade amostral, tomada exatamente para representar o desenvolvimento da palmeira desde a implantação no campo até plantas próximas do estádio de reprodução, há ainda a grande variabilidade genética do material atualmente disponível ao agricultor. Em experimentos conduzidos a campo, observa-se que o coeficiente de variação foi sempre elevado para os diferentes caracteres avaliados, estando entre 21 e $81 \%$, mesmo para plantas da mesma origem e idades (Chalá, 1993; Ares, et al. 2002a).

As etapas de pesagem para a obtenção da fitomassa com base em matéria seca tornaram evidente que o teor de água da planta como um todo e de seus diferentes componentes é altíssimo, variando de $67,3 \%$ para as folhas a $91,4 \%$ para o estipe e o palmito, com média geral de 79,3\% de umidade. Há uma pequena variação no teor de umidade da planta em função do tamanho da mesma. Plantas menores têm maior teor de água nas folhas que plantas maiores (69,9\% para plantas $<1,5 \mathrm{~m}$ e $65,5 \%$ para plantas $>1,5 \mathrm{~m}$ ). Não obstante, quando se compara o teor total de umidade na planta, nota-se que a umidade é maior em plantas mais altas $(78,1 \%$ para plantas $<1,5 \mathrm{~m}$ e $80,1 \%$ para plantas $>1,5 \mathrm{~m}$ ). Isso ocorre devido a maior contribuição do palmito e do estipe macio

Foram testadas diferentes equações para o cálculo da estimativa de fitomassa da pupunheira em relação à área foliar, número de folhas, altura e perímetro, observando-se que a variável com pior ajuste foi sempre o número de folhas. $\mathrm{O}$ melhor ajuste, expresso comparativamente na figura 1 para todas as variáveis, foi obtido quando do uso da área foliar, com coeficiente de determinação elevado $\left(\mathrm{R}^{2}=0,98\right)$. Resultados semelhantes foram obtidos por Szott et al. (1993) ao estimar a biomassa de pupunheira adulta. No entanto, a estimativa da área foliar é obtida de forma indireta, demandando análises destrutivas a campo e trabalho considerável, posteriormente executado em laboratório. Estimativas do índice de área foliar, e conseqüentemente da área foliar, mais diretas e sem necessidade de análises destrutivas, têm sido obtidas por meio da transmitância da radiaao longo do tempo. ção a vários ângulos, usando-se equipamento apropriado (Lamade, 1997; Hicks e Lascano, 1995; Weles e Norman, 1991). Não obstante, há necessidade de validação do procedimento usando-se métodos tradicionais, de natureza destrutiva, ainda que seja em amostras pequenas (Welles, 1990; Eschenbach e Kappen, 1996).

Considerando as variáveis de mais fácil mensuração, concluiu-se que a altura foi a que apresentou melhor correlação com a fitomassa. A estimativa da fitomassa área das plantas-mãe com base na altura variou de 0,02 a 21,20 kg, (média de 2,44 kg, DP de 2,91 kg, CV de $118,88 \%$, IC de $0,64 \mathrm{~kg}$ ), e esteve entre 0,01 a $0,08 \mathrm{~kg}$ (média de $0,02 \mathrm{~kg}$, DP de $0,02 \mathrm{~kg}$, IC de $0,01 \mathrm{~kg}$ ) para os perfilhos. Os coeficientes de determinação da fitomassa aérea, tendo a altura como variável independente, mostraram sempre maior magnitude do que a mesma estimativa obtida por meio do perímetro. A maior utilidade da variável altura, em comparação à variável diâmetro ou perímetro, para expressar respostas de crescimento e produção da pupunheira visando a produção de palmito, já tinha sido anteriormente reportada por Bovi et al. (1993a) utilizando pupunheiras adultas da mesma raça (Yurimaguas) aqui estudada.

Dentre todas as equações testadas que utilizam a altura para a estimativa da fitomassa, a descrita na figura 1 $\left(\mathrm{R}^{2}=0,89\right)$ foi a que apresentou menor número de parâmetros (a e b) e, quando comparada com o perímetro $\left(\mathrm{R}^{2}=0,76\right)$, melhor distribuição dos dados na curva. Tais resultados são discordantes dos reportados recentemente por Ares et al. (2002a), para os quais o uso do diâmetro da planta foi a variável que melhor se ajustou para calcular a biomassa de pupunheiras com 1,9 a 21 anos em cultivo na Costa Rica. Não obstante, os autores reconhecem que a melhor equação $\left(\mathrm{y}=\mathrm{a}^{*} \mathrm{x}^{\mathrm{b}}, \mathrm{R}^{2}=0,85\right)$, por eles reportada, superestimou a biomassa total (e dos componentes desta) em plantas compreendidas entre a fase do estabelecimento a campo e a fase de rápido crescimento. A experiência mostra que a superestimativa pode ser devido a uma série de fatores, dentre eles, as dificuldades de se medir o diâmetro na altura 
convencionada no estudo supracitado (5 $\mathrm{cm})$; diferentes profundidades de plantio; variação da medida em função da senescência das folhas; e erros na tomada da medida, visto que a haste da pupunheira não é perfeitamente cilíndrica. Já a altura pode ser medida com maior precisão, desde que se utilizem os critérios reportados por Clement e Bovi (2000).

Estabelecida uma comparação gráfica 1:1 dos dados observados com os calculados das equações anteriormente mencionadas (gráficos não apresentados), observa-se que, de uma forma geral, as equações que utilizam a altura como variável independente são as que têm melhor aproximação aos dados reais $\left(\mathrm{R}^{2}=0,91\right)$, especialmente para as alturas até 3,0 m, tamanho máximo para o corte econômico do palmito. Quando a variável independente é o perímetro da planta, os dados observados e os estimados encontram-se muito dispersos. Por sua vez, quando do uso da área foliar como variável independente para se estimar a fitomassa observa-se um grau de determinação bastante elevado $\left(\mathrm{R}^{2}=\right.$ $0,99)$, o que sugere a grande precisão do uso da área foliar para esse cálculo. No entanto, como discutido anteriormente, esse ainda é um parâmetro de difícil medição, tornando pouco prática, e bastante demorada, a sua obtenção rotineira. Devido ao alto ajuste obtido quando do uso da altura da haste principal (em comparação com o perímetro), essa seria a medida direta mais rápida de ser obtida e mais adequada a ser empregada em trabalhos relacionados ao cultivo da pupunheira para produção comercial de palmito.

A contribuição dos perfilhos para a fitomassa total durante a primeira fase da cultura (plantio até a primeira colheita de palmito) é bastante pequena, como pode ser visualizada na figura 2. Plantas com 12 meses a campo possuíam altura da haste principal variando de 0,14 a 2,16 m, fitomassa aérea estimada da haste principal entre 0,25 a $2,88 \mathrm{~kg}$ e fitomassa aérea dos perfilhos entre $0 \mathrm{e}$ $0,07 \mathrm{~kg}$. A fitomassa aérea total (haste principal e perfilhos) variou de 0,25 a $2,95 \mathrm{~kg}$ por planta, correspondente a 1,20 a $14,25 \mathrm{~kg}$ por planta de massa fresca.

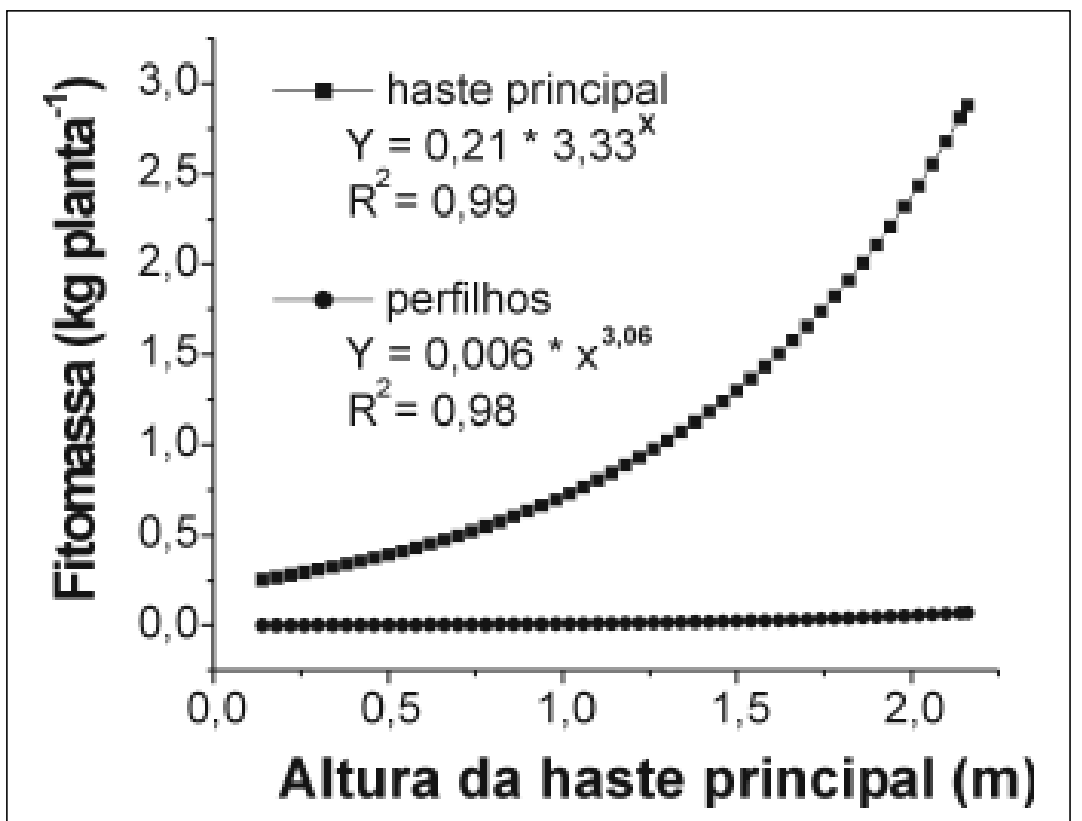

Figura 2. Comparação gráfica entre a fitomassa $\left(\mathrm{kg} \mathrm{planta}^{-1}\right) \mathrm{da}$ haste principal (planta mãe) e dos perfilhos, em função da altura (do solo até a inserção da folha +1 ) de pupunheiras com 12 meses a campo. Ubatuba, IAC, 2002.

A grande dispersão dos dados mostra a variabilidade genética ainda presente no material disponível ao agricultor e o nível acentuado de resposta aos tratamentos impostos apresentados pela pupunheira. A contribuição dos perfilhos para a fitomassa aérea total, ainda que bastante pequena e desuniforme (considerando plantas individuais), vai aumentando à medida que a planta cresce. No experimento em estudo, foi observado que em plantas com até $50 \mathrm{~cm}$ de altura da haste principal, a fitomassa dos perfilhos representava apenas $0,48 \%$ da fitomassa total. No intervalo entre $51 \mathrm{e}$ $100 \mathrm{~cm}$ de altura observou-se uma contribuição de $0,76 \%$, passando para $1,17 \%$ no intervalo 101 a $150 \mathrm{~cm}$. Pupunheiras nas quais a haste principal media de 151 a $216 \mathrm{~cm}$ de altura, e que correspondem à faixa de plantas com hastes aptas para colheita de palmito, apresentaram fitomassa dos perfilhos estimada em apenas $2,03 \%$ da fitomassa total. Tal fato sugere que a dominância da planta-mãe é muito grande até esse estádio de desenvolvimento e indica que, em plantas com até 2,16 metros de altura, estimando-se a fitomassa aérea apenas da haste principal, obtém-se uma precisão adequada da fitomassa aérea total da touceira.

\section{LITERATURA CITADA}

ARES, A.; FOWNES, J.H. Productivity, nutrient and water-use efficiency of Eucalyptus saligna and Toona ciliata in Hawaii. Forest Ecology and Management, v.139, n.2, p.227-236, 2000

ARES, A.; BONICHES, J.; MOLINA, E.; YOST, R.S. Bactris gasipaes agroecosystems for heart-ofpalm production in Costa Rica: change in biomass, nutrient and carbon pools with stand age and plant density. Field Crops, v.74, n.1, p.13-22, 2002a.

ARES, A.; QUESADA, J.P.; BONICHE, J.; YOST, R.S.; MOLINA, E.; SMYTH, J. Allometric relationships in Bactris gasipaes for heart-of-palm production agroecosystems in Costa Rica. Journal of Agricultural Science, v.138, n.3, p.285-292, 2002 b. BENINCASA, M.M.P. Analise de crescimento de plantas, Jaboticabal, FUNEP, 1988, 42 p.

BOVI, M.L.A. Palmito pupunha informações básicas para cultivo, Campinas, IAC - Boletim Técnico 173, 1998a, 50 p.

BOVI, M.L.A. Expansão do cultivo da pupunheira para palmito no Brasil. Horticultura Brasileira, Brasília, v.15 (supl.), p.183-185, 1998 b.

BOVI, M.L.A.; GODOY JR., G.; CAMARGO, S.B.; SPIERING, S.H. Caracteres indiretos na seleção de pupunheiras inermes (Bactris gasipaes H.B.K.) para palmito. In: CONGRESO INTERNACIONAL SOBRE BIOLOGIA, AGRONOMIA E INDUSTRIALIZACION DEL PIJUAYO, 4., 1991, Iquitos. Anais. San José: UFCR, 1993a. p.163-176. CHALÁ, V.H. Evaluación de 8 densidades de siembra de Bactris gasipaes H.B.K. para la producción de palmito en la región amazonica ecuatorial. In: CONGRESO INTERNACIONAL SOBRE BIOLOGIA, AGRONOMIA E INDUSTRIALIZACION DEL PIJUAYO, 4., 1991, Iquitos. Anais. San José: UFCR, 1993. p.255-265. 
CLEMENT, C.R. Growth and analysis of pejibaye (Bactris gasipaes Kunth, Palmae) in Hawaii. Honolulu, Honolulu: University of Hawaii, 1995 , 221 p. (Tese doutorado)

CLEMENT, C.R.; BOVI, M.L.A. Padronização de medidas de crescimento e produção em experimentos com pupunha para palmito. Acta Amazônica, v.30, n.3, p.349-362, 2000.

CLEMENT, C.R.; CAMPOS, J.K.P.; PLÁCIDO NETO, J.J. Estimación de la biomasa de la hoja del pejibaye (Bactris gasipaes H.B.K.). Revista de Biologia Tropical, v.38, n.2, p.395-400, 1990. ESCHENBACH, C.; KAPPEN, L. Leaf area index determination in an alder forest: a comparison of three methods. Journal of Experimental Botany, v.47, n.302, p.1457-1462, 1996.

FERREIRA, V.L.P.; MIYA, E.E.; SHIROSE, I.; ARANHA, C.; SILVA, E.A.M.; HIGHLANDS, M.E. Comparação físico-químico-sensorial do palmito de três espécies de palmeira. Coletânea do ITAL, v.7, n.2, p.389-416, 1976.
HICKS, S.K.; LASCANO, R.J. Estimation of leaf area index for cotton canopies using the LI-COR LAI-2000 plant canopy analyzer. Agronomy Journal, v.87, n.3, p.458-464, 1995.

HYAMS, D. CurveExpert 1.3: A comprehensive curve fitting system for Windows. http:// wwwebicom.net/ dhyams. Starkville, 1997. [software].

JONGSCHAAP, R. Palmito (Bactris gasipaes H.B.K.) growth and management in the humidlowlands of the Atlantic zone of Costa Rica. Atlantic Zone Programme Report no. 60 (Field Report no. 107). Agric. Univ. Wageningen, CATIE and Ministerio de Agricultura y Ganaderia, Costa Rica, 1993. 52 p.

KIRA, T.; SHIDEI, R. Primary production and turnover of organic matter in different forest ecosystems of the western Pacific. Japanese Journal of Ecology, v.17, n.1, p.70-87, 1967.

LAMADE, E. Métode rapide de mesure de l'indice foliaire du palmier à huile. Plantation, recherche, développement, v. 4, n. 6, p.385-391, 1997.
LUCCHESI, A.A. Fatores da produção vegetal, In: CASTRO P.R.C.; FERREIRA S.O.; YAMADA T. (Eds.), Ecofisiologia da produção agrícola, POTAFOS, Piracicaba SP., 1987, 249 p.

STEEL, R.G.; TORRIE, J.H. Principles and producedures of statistics. New York, MacGrawHill. 1980, $632 \mathrm{p}$

SZOTT L.T.; AREVALO L.; PEREZ J., Allometric relationships in pijuayo (Bactris gasipaes H.B.K.). In: CONGRESO INTERNACIONAL SOBRE BIOLOGIA, AGRONOMIA E INDUSTRIALIZACION DEL PIJUAYO, 4., 1991, Iquitos. Anais. San José: UFCR, 1993, p.91114.

VEGA, F.V.A. Uso de lodo de esgoto na implantação da cultura da pupunheira (Bactris gasipaes Kunth). Campinas: Instituto Agronômico de Campinas, 2003, 126 p. (Dissertação mestrado)

WELLES, J.M. Some indirect methods of estimating canopy structure. Remote Sensing Reviews, v.5, n.1, p.31-43, 1990.

WELLES, J.M.; NORMAN, J.M. Instrument for indirect measurement of canopy arquitecture. Agronomy Journal, v.83, n.4, p.818-825, 1991. 